\title{
Successful hematopoietic stem cell transplantation in a patient with congenital dyserythropoietic anemia type II
}

\author{
Unal S, Russo R, Gumruk F, Kuskonmaz B, Cetin M, Sayli T, Tavil B, \\ Langella C, Iolascon A, Cetinkaya DU. Successful hematopoietic stem \\ cell transplantation in a patient with congenital dyserythropoietic \\ anemia type II.
}

Abstract: CDA are a group of inherited, rare diseases that are characterized by dyserythropoiesis and ineffective erythropoiesis associated with transfusion dependency in approximately $10 \%$ of cases. For these latter patients, the only curative treatment is HSCT. There are very limited data on HSCT experience in this rare disease. Herein, we report a five-yr six-month-old girl with compound heterozygous mutations in $S E C 23 B$ gene, who was diagnosed to have CDA type II and underwent successful HSCT from her matched sibling donor.

\author{
Sule Unal ${ }^{1}$, Roberta Russo ${ }^{2,3}$, Fatma \\ Gumruk ${ }^{1}$, Baris Kuskonmaz ${ }^{1}$, Mualla \\ Cetin $^{1}$, Tulin Sayli ${ }^{4}$, Betul Tavil ${ }^{1}$, \\ Concetta Langella ${ }^{2,3}$, Achille Iolascon ${ }^{2,3}$ \\ and Duygu Uckan Cetinkaya ${ }^{1}$ \\ ${ }^{1}$ Division of Pediatric Hematology, Hacettepe \\ University, Ankara, Turkey, ${ }^{2}$ Department of \\ Molecular Medicine and Medical Biotechnologies, \\ University Federico II, Naples, Italy, ${ }^{3}$ CEINGE \\ Advanced Biotechnologies, Naples, Italy, ${ }^{4}$ Ankara \\ Pediatric Hematology and Oncology Research \\ Hospital, Ankara, Turkey \\ Key words: transplantation - congenital \\ dyserythropoietic anemias - iron - SEC23B \\ Sule Unal, Division of Pediatric Hematology, \\ Hacettepe University, Ankara 06100, Turkey \\ Tel.: +90 3123051170 \\ Fax: +90 3123112398 \\ E-mail: suleunal@hacettepe.edu.tr \\ Accepted for publication 24 February 2014
}

CDA are a group of rare heterogenous disorders characterized by dyserythropoiesis, ineffective erythropoiesis, iron overload, and specific light and electron microscopy findings of nucleated erythroid precursors (1-3). Patients usually present with anemia, jaundice, splenomegaly, low reticulocyte count despite erythroid hyperactivity (3). There are broadly three main types of CDA (CDA I, II, and III), due to mutations in $C D A N 1, S E C 23 B$, and KIF23, respectively (3-7). However, there are additional CDA variants that do not fit to any three classical types, such as CDA patients with KLFI and GATAI mutations. To date, 157 cases from 137 different CDA II families with $S E C 23 B$ mutations were molecularly analyzed $(3,8)$. Type II patients are charac-

Abbreviations: ATG, antithymocyte globulin; CDA, congenital dyserythropoietic anemias; CMV, cytomegalovirus; CyA, cyclosporin A; gDNA, genomic DNA; GVHD, graft-versus-host disease; HSCT, hematopoietic stem cell transplantation; LDH, lactate dehydrogenase; MCV, mean corpuscular volume; RDW, red cell distribution width; VOD, veno-occlusive disease. terized by erythroid hyperactivity in bone marrow with no megaloblastic changes, in addition to high numbers of binucleated normoblasts with occasional multinucleated erythroid precursors (1). The nucleated erythroid precursors of patients with CDA type II may exhibit double membrane appearance under electron microscopy (9). There are patients with CDA who have mild-to-moderate anemia who require no regular transfusions. On the other hand, treatment alternatives include erythrocyte transfusions for patients with severe anemia, iron chelation to decrease complications related to transfusional iron overload, interferon alpha for some of type I and splenectomy for some of the type II patients $(10,11)$. However, the only curative treatment for patients with CDA is HSCT (12, 13). There is scarce data in the reported literature on the use of HSCT as a therapeutic and curative option in patients with CDA $(2,12,14-17)$. Herein, we report a five-yr six-month-old girl with CDA II who had compound heterozygous mutations in SEC23B gene and was successfully transplanted from a matched sibling donor. 


\section{Case report}

A five-yr six-month-old girl was referred to our center for HSCT after being followed up under a chronic transfusion program, since 40 days of age. She had a history of initially monthly erythrocyte transfusions which progressed to every 23 wk over the last two yr. She was on folic acid supplementation and on iron chelation with desferrioxamine for one yr.

The family history revealed no consanguinity and she had one healthy brother. The physical examination revealed hepatosplenomegaly, subcostally $3 \mathrm{~cm}$ and $8 \mathrm{~cm}$ palpable, respectively. She had no skeletal abnormalities. The hemogram at presentation to our center revealed: $\mathrm{Hb} 8.9 \mathrm{~g} / \mathrm{dL}$, RBC $3.10 \times 10^{6} / \mu \mathrm{L}$, Hct $26.1 \%$, MCV $84 \mathrm{fL}$, RDW 14.1 , WBC $10.3 \times 10^{9} / \mathrm{L}$, platelet $519 \times$ $10^{9} / \mathrm{L}$. Reticulocyte count was $2.4 \%$ (absolute count 74 400) and peripheral blood smear revealed anisocytosis and poikilocytosis. The liver and kidney functions were within normal limits. Serum total and indirect bilirubin levels were 0.99 and $0.71 \mathrm{mg} / \mathrm{dL}$, respectively. Serum $\mathrm{LDH}$ was $545 \mathrm{IU} / \mathrm{L}$, and serum ferritin was measured as $2030 \mathrm{ng} / \mathrm{mL}$. She was immune to HBV and negative for HCV and HIV. The cardiac iron load was evaluated with T2* MRI and revealed T2* above $20 \mathrm{~ms}$, indicating no iron load. Left ventricular ejection fraction was $73 \%$. Liver biopsy revealed dilatation in the portal areas, fine fibrous septation in addition to severe iron load in hepatocytes and Kupffer cells. The Ham test was found negative that may partially be attributed to recent transfusion history. The bone marrow examination under light microscopy revealed erythroid hyperactivity and of these normoblasts one-third were double-nucleated cells, with very few multinucleated cells as well. Megaloblastic normoblasts were present, but no nuclear bridging was noted. In the electron microscopy of the bone marrow nuclear protrusions, multinuclearity, karyorrhexis, excessive membrane structures forming invaginations or cisternae, and encompassing the circumference of the cell were seen in varying degrees in the majority of the normoblasts. A clinical diagnosis of CDA type II was established after exclusion of other causes associated with dyserythropoiesis and ineffective erythropoiesis, including thalassemia syndromes, vitamin B12 and folate deficiencies, myelodysplastic syndrome, and sideroblastic anemia. HSCT was performed from the HLA 6/6-matched brother after conditioning with busulfan $(12.8 \mathrm{mg} / \mathrm{kg} /$ total dose, iv, $0.8 \mathrm{mg} / \mathrm{kg} / \mathrm{dose}$, qid, for four days, from day -10 to -7$)$, cyclophosphamide $(200 \mathrm{mg} / \mathrm{kg} /$ total dose, $50 \mathrm{mg} / \mathrm{kg} /$ day, for four days, from day -5 to -2 , with mesna infusions), and ATG $(10 \mathrm{mg} / \mathrm{kg} /$ day, for three days, from -4 to -2 ). GVHD prophylaxis consisted of CyA (iv $3 \mathrm{mg} / \mathrm{kg} / \mathrm{day}$, initiated on day -1 , and changed to oral form when adequate nutrition was sustained) plus methotrexate (iv, $10 \mathrm{mg} / \mathrm{m}^{2}$, on days $+1,+3,+6$ days). Prophylaxis for VOD was made by ursodeoxycholic acid $(20 \mathrm{mg} / \mathrm{kg} / \mathrm{day}$, bid, till +28 th day) and enoxaparin $(1 \times 0.8 \mathrm{mg} / \mathrm{kg} /$ day, subcutaneously, till +28 th day). Bone marrow was used as stem cell source without G-CSF mobilization. Erythrocyte depletion by hetastarch sedimentation was carried out due to major ABO incompatibility. The nucleated cell dose infused was $9.4 \times 10^{8} / \mathrm{kg}$, and the $\mathrm{CD} 34^{+}$cell dose was $4.7 \times 10^{6} / \mathrm{kg}$. Neutrophil and platelet engraftments were observed on the 21 st and 30th days of transplantation, respectively. The patient developed grade III mucositis and neutropenic fever that were managed successfully. The pre-emptive screening for CMV was made on a twice weekly schedule after neutrophil engraftment using CMV DNA measurement by quantitative PCR. Antifungal prophylaxis was made by triflucan $(5 \mathrm{mg} / \mathrm{kg} /$ day, once daily, iv, between +1 st to +30 th days), and antiviral prophylaxis was provided by acyclovir $\left(500 \mathrm{mg} / \mathrm{m}^{2} /\right.$ day, bid, between +1 st to +30 th days). She had no CMV activation, VOD, acute or chronic GVHD during the follow-up and was discharged on the 33rd day of HSCT. The patient had consistently full donor chimerism and was initiated on iron chelation with deferasirox at post-transplant $+2 \mathrm{yr}$. She is currently $12 \mathrm{yr}$ old and has been transfusion independent throughout the postHSCT duration of six and a half yr. Sequencing analyses on pre- and post-HSCT gDNA samples were performed as previously described (5). The molecular analyses from stored pre-HSCT gDNA revealed double heterozygous missense mutations in SEC23B gene (c.1489C>T, p.R497C in the exon 13 and c. $1588 \mathrm{C}>\mathrm{T}$, p.R530W in the exon 14), already described as causative (1). Sequencing analyses from after HSCT gDNA revealed the almost complete absence of both mutated alleles, indicating that the donor was not heterozygous (Fig. 1). The family did not consent for the molecular study of the healthy brother.

\section{Discussion}

The application of HSCT in a patient with CDA has some challenges including the possible allosensitization related to the previous transfusions, transfusional iron overload that may increase the toxicities related to conditioning regimen, and being an autosomal recessively inherited disease finding a healthy matched sibling donor. In CDA 

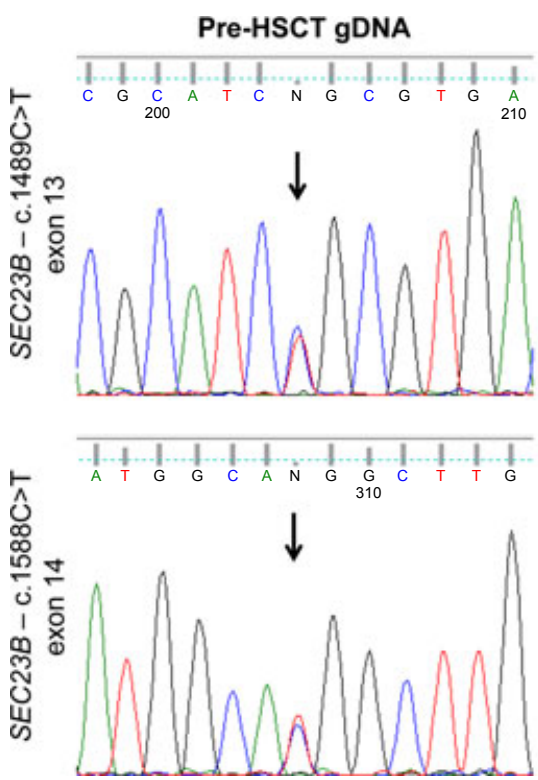
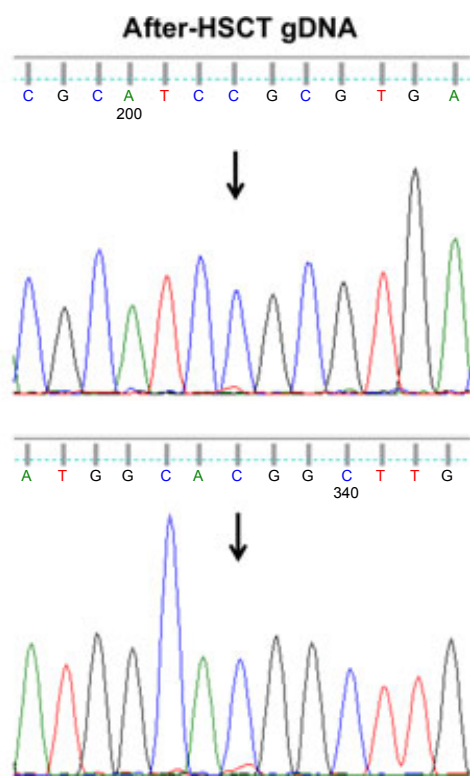

Fig. 1. gDNA molecular analyses pre- and post-HSCT. Analysis of both $S E C 23 B$ mutations highlighted the presence of a double peak (heterozygous state) in the electropherograms from preHSCT gDNA of the proband. Conversely, after HSCT gDNA sequencing just showed the presence of the wild-type allele nucleotides. Mutation quantification performed by Mutation Surveyor software showed a small residual percentage of mutated alleles in after HSCT gDNA $(7.2 \%)$ compared with mutated alleles in pre-HSCT gDNA $(50.6 \%)$.
II patients, splenectomy may be a therapeutic but not curative option in some of the patients (11). Our patient had a long history of erythrocyte transfusions, and splenectomy was not performed due to the possibility of unresponsiveness to splenectomy and to prevent lifelong risks of sepsis and pulmonary hypertension.

Our patient received iron chelation with desferrioxamine for only one yr prior to HSCT. According to pre-HSCT risk assessment by Lucarelli Classification system that has originally been used for thalassemic patients, the risk of HSCT in thalassemic patients was determined by the presence of hepatomegaly, portal fibrosis, and irregular chelation history (18). According to this classification system, our patient was assigned as Class III. In thalassemic patients with Class III disease, Protocol 26 has been suggested which includes transfusion with target $\mathrm{Hb}$ of 14 $15 \mathrm{~g} / \mathrm{dL}$, iv desferrioxamine, hydroxyurea, azathioprine, G-CSF, erythropoietin, fludarabine, and busulfan (19). This regimen improved the overall survival and thalassemia-free survival to $93 \%$ and $85 \%$, respectively, in Class III thalassemic patients (19).

On the other hand, there is limited data on the conditioning regimen preferences of patients with CDA. Of the previously reported patients with CDA who underwent HSCT, one patient with serum ferritin level of $3500 \mathrm{ng} / \mathrm{mL}$ has been transplanted after conditioning with busulfan, thiotepa, and fludarabine and was reported to be alive for 36 months after HSCT. This patient was reported to be placed on phlebotomy program after HSCT (14). Another patient of 13 months old, who had hydrops fetalis and transfused regularly beginning from the intrauterine period has been transplanted by the end of the first year after conditioning with busulfan and cyclophosphamide (15). Ayas et al. reported three patients with CDA type I who have undergone HSCT and all alive for a follow-up period of five, two, and two and a half yr, respectively, after conditioning with busulfan, cyclophosphamide, and ATG (2). Another five-yr-old patient with CDA has been reported to need second transplantation after initial conditioning with busulfan and cyclophosphamide, and the second HSCT was conditioned with busulfan, cyclophosphamide, melphalan, and ATG. The patient has been reported as alive and transfusion independent after second transplantation (16). Besides these six patients reported by four centers who were transplanted from HLA-matched sibling donors $(2,14-16)$, there are two recent reports of successful HSCT in patients with CDA type II from HLA-matched unrelated donors $(12,17)$. The first patient who underwent unrelated HSCT was conditioned intensively with azathioprine, fludarabine, busulfan, thiotepa, and cyclophosphamide (12). In a very recent report, an 11-month-old patient with CDA II, who presented with severe fetal hydrops and required intrauterine transfusions, was transplanted from a matched unrelated donor after conditioning with busulfan, cyclophosphamide, and melphalan (17). This patient received ATG and CyA for GVHD prophylaxis (17). According to our experience and review of the literature $(2,14-16)$, in HSCT from a matched sibling donor, conditioning with busulfan, cyclophosphamide and ATG is a safe and effective 
option even in patients with CDA risk classified as Class III, according to Lucarelli Classification (18). This conditioning was well tolerated in our patient.

ATG has been used in the conditioning regimen in order to reduce the risk of graft rejection, particularly in patients who were transfused heavily prior to HSCT. Additionally, ATG has been reported to decrease the risk of GVHD and increase the overall survival among patients with thalassemia major who underwent HSCT (20). Additionally, the dose of ATG has been compared in a previous study among patients who underwent HSCT, and there were no differences in terms of acute and chronic GVHD between patients who received ATG 30 vs. $60 \mathrm{mg} / \mathrm{kg} /$ total doses, and transplant-related mortality was found lower in $30 \mathrm{mg} / \mathrm{kg}$ ATG receivers, and the difference was attributed to increased infections among higher dose receivers (21).

In conclusion, our knowledge about the HSCT experience in patients with CDA is limited and the conditioning regimens vary widely. In our patient, busulfan, cyclophosphamide, and ATG regimen was well tolerated, although being a high-risk patient with hepatomegaly, poor chelation history, and fibrosis findings in liver biopsy. Considering the difficulties in diagnosis of CDA, as it is a diagnosis of exclusion, the increased use of molecular testing for diagnostic purposes will increase the number of patients diagnosed which will in turn increase the HSCT rates with this indication.

\section{Acknowledgments}

This work was supported by grants from the Italian Ministero dell'Universitá e della Ricerca, by grants MURPS 35-126/Ind, by grants from Regione Campania (DGRC2362/07), by EU Contract LSHM-CT-2006-037296, Italian Telethon Foundation Grant GGP 09044 to AI, Rome, Italy.

\section{Conflict of interest}

There is no conflict of interest.

\section{Authors' contributions}

All authors contributed equally.

\section{References}

1. Iolascon A, Russo R, Delaunay J. Congenital dyserythropoietic anemias. Curr Opin Hematol 2011: 18: 146-151.

2. Ayas M, Al-Jefri A, Baothman A, et al. Transfusion-dependent congenital dyserythropoietic anemia type I successfully treated with allogeneic stem cell transplantation. Bone Marrow Transplant 2002: 29: 681-682.
3. Iolascon A, Heimpel H, Wahlin A, Tamary H. Congenital dyserythropoietic anemias: Molecular insights and diagnostic approach. Blood 2013: 122: 2162-2166.

4. Dgany O, Avidan N, Delaunay J, et al. Congenital dyserythropoietic anemia type I is caused by mutations in codanin-1. Am J Hum Genet 2002: 71: 1467-1474.

5. Schwarz K, Iolascon A, Verissimo F, et al. Mutations affecting the secretory COPII coat component SEC23B cause congenital dyserythropoietic anemia type II. Nat Genet 2009: 41: 936-940.

6. Bianchi P, Fermo E, Vercellati C, et al. Congenital dyserythropoietic anemia type II (CDAII) is caused by mutations in the SEC23B gene. Hum Mutat 2009: 30: 1292-1298.

7. Liljeholm M, Irvine AF, Vikberg AL, et al. Congenital dyserythropoietic anemia type III (CDA III) is caused by a mutation in kinesin family member, KIF23. Blood 2013: 121: 4791-4799.

8. Iolascon A, Esposito MR, Russo R. Clinical aspects and pathogenesis of congenital dyserythropoietic anemias: From morphology to molecular approach. Haematologica 2012: 97: 1786-1794.

9. Alloisio N, Texier P, Denoroy L, et al. The cisternae decorating the red blood cell membrane in congenital dyserythropoietic anemia (type II) originate from the endoplasmic reticulum. Blood 1996: 87: 4433-4439.

10. Goede JS, Benz R, Fehr J, Schwarz K, Heimpel H. Congenital dyserythropoietic anemia type I with bone abnormalities, mutations of the CDAN I gene, and significant responsiveness to alpha-interferon therapy. Ann Hematol 2006: 85: 591-595.

11. Iolascon A, Delaunay J, Wickramasinghe SN, et al. Natural history of congenital dyserythropoietic anemia type II. Blood 2001: 98: 1258-1260.

12. Buchbinder D, Nugent D, Vu D, et al. Unrelated hematopoietic stem cell transplantation in a patient with congenital dyserythropoietic anemia and iron overload. Pediatr Transplant 2012: 16: E69-E73.

13. Renella R, Wood WG. The congenital dyserythropoietic anemias. Hematol Oncol Clin North Am 2009: 23: 283-306.

14. Iolascon A, Sabato V, de Mattia D, Locatelli F. Bone marrow transplantation in a case of severe, type II congenital dyserythropoietic anaemia (CDA II). Bone Marrow Transplant 2001: 27: 213-215.

15. Remacha AF, Badell I, Pujol-Moix N, et al. Hydrops fetalis associated congenital dyserythropoietic anemia treated with intrauterine transfusions and bone marrow transplantation. Blood 2002: 100: 356-358.

16. Ariffin WA, Karnaneedi S, Choo KE, et al. Congenital dyserythropoietic anaemia: Report of three cases. J Paediatr Child Health 1996: 32: 191.

17. Braun M, Wölfl M, Wiegering V, et al. Successful treatment of an infant with CDA type II by intrauterine transfusions and postnatal stem cell transplantation. Pediatr Blood Cancer 2014: 61: 743-745.

18. Lucarelli G, Galimberti M, Polchi P, et al. Bone marrow transplantation in patients with thalassemia. N Engl J Med 1990: 322: 417-421.

19. Sodani P, Gaziev D, Polchi P, et al. New approach for bone marrow transplantation in patients with class 3 thalassemia aged younger than 17 years. Blood 2004: 104: 1201-1203.

20. Bacigalupo A. Antilymphocyte/thymocyte globulin for graft versus host disease prophylaxis: Efficacy and side effects. Bone Marrow Transplant 2005: 35: 225-231.

21. Ayuk F, Diyachenko G, Zabelina T, et al. Comparison of two doses of antithymocyte globulin in patients undergoing matched unrelated donor allogeneic stem cell transplantation. Biol Blood Marrow Transplant 2008: 14: 913-919. 